\title{
Physical therapy plus general practitioners' care versus general practitioners' care alone for sciatica: a randomised clinical trial with a 12-month follow-up
}

\author{
Pim A. J. Luijsterburg • Arianne P. Verhagen • \\ Raymond W. J. G. Ostelo · Hans J. M. M. van den Hoogen • \\ Wilco C. Peul · Cees J. J. Avezaat · Bart W. Koes
}

Received: 2 February 2007/Revised: 25 September 2007/ Accepted: 2 December 2007/Published online: 3 January 2008

(C) The Author(s) 2007

\begin{abstract}
A randomised clinical trial in primary care with a 12-months follow-up period. About 135 patients with acute sciatica (recruited from May 2003 to November 2004) were randomised in two groups: (1) the intervention group received physical therapy (PT) added to the general practitioners' care, and (2) the control group with general practitioners' care only. To assess the effectiveness of PT additional to general practitioners' care compared to general practitioners' care alone, in patients with acute sciatica. There is a lack of knowledge concerning the effectiveness of PT in patients with sciatica. The primary outcome was patients' global perceived effect (GPE). Secondary
\end{abstract}

P. A. J. Luijsterburg $(\bowtie)$ · A. P. Verhagen · B. W. Koes Department of General Practice, Erasmus MC, PO Box 2040, 3000 CA Rotterdam, The Netherlands

e-mail: pimluijsterburg@home.nl

R. W. J. G. Ostelo

VU, Institute for Research in Extramural Medicine,

Amsterdam, The Netherlands

R. W. J. G. Ostelo

Amsterdam School of Allied Health Education,

Amsterdam, The Netherlands

H. J. M. M. van den Hoogen

General Practice, Asten, The Netherlands

W. C. Peul

Neurosurgery, Leids University Medical Center,

Leiden, The Netherlands

W. C. Peul

Neurosurgery, Medical Center Haaglanden,

Den Haag, The Netherlands

C. J. J. Avezaat

Neurosurgery, Erasmus MC, Rotterdam, The Netherlands outcomes were severity of leg and back pain, severity of disability, general health and absence from work. The outcomes were measured at 3, 6, 12 and 52 weeks after randomisation. At 3 months follow-up, $70 \%$ of the intervention group and $62 \%$ of the control group reported improvement (RR 1.1; 95\% CI 0.9-1.5). At 12 months follow-up, $79 \%$ of the intervention group and $56 \%$ of the control group reported improvement (RR 1.4; 95\% CI 1.1; 1.8). No significant differences regarding leg pain, functional status, fear of movement and health status were found at short-term or long-term follow-up. At 12 months follow-up, evidence was found that PT added to general practitioners' care is only more effective regarding GPE, and not more cost-effective in the treatment of patients with acute sciatica than general practitioners' care alone. There are indications that PT is especially effective regarding GPE in patients reporting severe disability at presentation.

Keywords Sciatica - Lumbosacral radicular syndrome . GP · Physical therapy $\cdot$ RCT

\section{Introduction}

The lumbosacral radicular syndrome (LRS), also called sciatica, is a disorder with radiating pain in the leg below the knee in one or more lumbar or sacral dermatomes, and can be accompanied by phenomena associated with nerve root tension or neurological deficits [18, 21, 22, 28]. A prolapsed disc is a frequent cause of LRS, but other causes include spinal or lateral recess stenosis, tumours and radiculitis $[7,21,22,28]$. The incidence of LRS in the Netherlands is estimated at 5 per 1,000 persons a year [7].

There is consensus that treatment of LRS in the first 6-8 weeks should be conservative. The exact content of the 
conservative treatment is, however not yet clear [25]. After the study of Vroomen et al. [26] bed rest is no longer regarded as a treatment option for LRS [16]. Assessed in a recent systematic review was the effectiveness of conservative treatments of LRS [15]. Thirty trials were included that evaluated injections $(n=14)$, traction $(n=9)$, physical therapy (PT) $(n=4)$, bed rest $(n=2)$, manipulation $(n=2)$, medication $(n=2)$ and acupuncture $(n=1)$ as a treatment for LRS. Because several trials indicated no evidence of an effect it is not recommended to use corticosteroid injections and traction as treatment option. Whether clinicians should prescribe PT, bed rest, manipulation or medication could not be concluded from this review. Therefore, there is no evidence that one type of conservative treatment is clearly superior to others for patients with LRS.

In an previous observational study it was shown that GPs did not adhere to the guideline regarding the referral to PT; almost half of patients with LRS were referred for PT, although this was not recommended in the guideline. No specific characteristic could be identified to explain this referral to PT [13]. Moreover, there is a lack of knowledge concerning the effectiveness of PT in LRS [15]. Therefore, this study aimed to assess the effectiveness of PT management additional to GP management compared to GP management alone, in patients with acute LRS.

\section{Methods}

More detailed information about the methods of the LRS trial is presented elsewhere [14]. The Erasmus Medical Center Ethics Committee approved the procedures and design of this trial.

\section{Study population}

Participating GPs in Rotterdam and the surrounding area invited patients with acute LRS to participate in the trial from May 2003 to November 2004. The GPs were invited by mail to participate in the trial. Table 1 shows the eligibility criteria.

The trial attempted to enrol 182 patients with LRS, 91 patients in both treatment groups. This sample size was regarded sufficient to detect a difference of $20 \%$ (with a $\alpha$ of 0.05 and a power of $80 \%$ ) in the primary outcome Global Perceived Effect (GPE) between the two treatment groups.

Randomisation

A concealed randomisation procedure [14] was used, which was based on a computer-generated randomisation
Table 1 Selection criteria for trial eligibility

Inclusion

Radiating (pain) complaints in the leg below the knee

Severity of complaints scored above 3 on an 11-point NRS

$(0=$ no complaints; $10=$ maximum complaints $)$

Duration of the (pain) complaints $<6$ weeks

Age between 18 and 65 years

Able to speak and read Dutch

Presence of one of the following symptoms

More pain on coughing, sneezing or straining

Decreased muscle strength in the leg

Sensory deficits in the leg

Decreased reflex activity in the leg

Positive straight leg raising test

Exclusion

Radiating (pain) complaints in the preceding 6 months

Back surgery in the past 3 years

Treated with epidural injections

Pregnancy

Co-morbidity that determines overall well-being

Direct indication for surgery (unbearable pain,

fast progression of paresis or cauda equina syndrome)

Expected loss to follow-up (i.e. moving to another part of the country, long-lasting foreign holiday)

list developed by an independent person. Concealment was ensured because patients' unique trial number was typed in a special database, which was not editable for the research assistant and a second randomisation action using the same trial number was not possible. Hereafter, the random allocation appeared on screen. In order to prevent unequal treatment group sizes, block randomisation was used with blocks of ten patients [19]. This means that after every tenth patient the number of patients allocated to both treatment groups was equal. The research assistant performed the randomisation after baseline measurement.

\section{Blinding}

For obvious reasons the GPs, physical therapists and patients were not blinded for treatment allocation. The statistical analysis and interpretation of the findings was audited and verified by an independent statistician.

\section{GP care}

All patients were treated by the GP according to their clinical guideline [21]. GPs gave information and advice about LRS and, if necessary, prescribed (pain) medication (see Fig. 1). 
Fig. 1 Summary of the clinical guideline 'Lumbosacral radicular syndrome' of the Dutch College of General Practitioners (1996)
Definition: The lumbosacral radicular syndrome: radiating pain and/or neurological deficits in one or more lumbar or sacral dermatomes, often associated with back pain; mostly caused by irritation and compression (traction) of the nerve root.

History taking: Ask about: 1) localisation, radiation, intensity and duration of the pain, 2) influence of rest, movement and posture, 3) development of the complaints, 4) interference with daily activities caused by leg pain, 5) decreased muscle strength and sensory deficits, 6 ) influence of coughing, sneezing or straining, 7) previous history of back complaints, and 8) urinary problems and saddle anaesthesia.

Physical examination: 1) physical inspection (spine and pelvis), 2) active examination (ante-, retro-, lateroflexion), and 3) Lasègue sign and test of Bragard. If there is a positive Lasègue sign, decreased muscle strength or sensory deficits perform: 4) ankle tendon reflex and knee tendon reflex, 5) sensory examination of the lateral and medial side of the foot, 6) muscular strength of the big toe, walking on heels and toes, and 7) crossed test of Lasègue.

Additional examinations: $\mathrm{X}$-rays should only be ordered in case of suspicion on malignancy or a fracture due to osteoporosis.

Evaluation: The lumbosacral radicular syndrome should be diagnosed if there are radiating complaints in the leg below the knee, plus one of the following findings: 1) a positive Lasègue sign (or Bragard), or 2) neurological deficits reducible to a single nerve root.

Information and advice: Explain to the patient that radiating complaints are caused by a prolapsed disc that gives pressure on a nerve in the back. There is a favourable coursein $80 \%$ of the patients with conservative care. Back pain may persist after the leg pain has gone.

Advice the patient to perform the usual daily activities but to avoid painful movements. Gradually increase the activities. Gradually increase the activities to normal level in six weeks and to patients' level in six to twelve weeks. Follow-up: Evaluate the effect of treatment by checking Lasègue sign and the severity of the complaints. Check patients with severe complaints daily and subsequently at least once a week. Accompany the patient till full resumption of daily activities.

Drug treatment: If desired: paracetamol (4-6 dd, 500mg), ibuprofen (3-4 dd, $400 \mathrm{mg}$ ), diclofenac (3-4 dd, 25-50 mg), or naproxen (2-3 dd, $250 \mathrm{mg})$.

Referral: Refer in an instant: 1) cauda equina syndrome, or 2) progressive paresis within a few days in spite of conservative care. Refer for diagnostics and judgement for indication for surgery: 1) Severe radicular pain in spite of bed rest and adequate medication, 2) Severe paresis or progressive paresis in spite of adequate care (walking on heels and toes is impossible), 3) doubtful diagnosis, or 4) mild complaints with no improvement after six to eight weeks.

\section{Physical therapy}

Physical therapy treatment consists of exercise therapy as well as giving information and advice about LRS. Passive modalities such as massage and manipulation techniques, or applications such as ultrasound therapy or electrotherapy were not allowed. The treatment protocol was developed in a consensus meeting with participating physical therapists. They acted as coaches and guided the patient in order to stimulate return to activity (type/content of the exercises was left to the expertise of the participating physical therapists), despite the pain experience. Both GP and PT interventions (only in an one to one setting; group settings were not allowed) were restricted to a maximum of nine treatments/consultations in the first 6 weeks after randomisation.

\section{Measurements}

Collected at baseline were patients' characteristics such as gender and date of birth. Standardised history taking was used to establish whether patients were familiar with LRS in the past, reported more pain in the leg on coughing/ sneezing or straining, on sitting, standing, walking and lying down and whether patients reported decreased muscle strength and sensory deficits in the leg. The physical examination included amongst others the straight leg raising test and the test of Bragard [9]. The primary outcome and most of the secondary outcomes were scored by the patients. At 3, 6, 12 and 52 weeks follow up the patients received a questionnaire by mail.

These questionnaires also measured absence from work due to LRS (in days) and medical consumption (i.e. medication use, additional therapies, visits to GP, PT or specialists).

\section{Primary outcome measure}

The primary outcome measure was the GPE, measured on a seven-point scale ranging from $1=$ completely recovered to $7=$ vastly worsened $[1,2,6]$. These ratings were dichotomised as improved ('completely recovered' and 'much improved'), versus not improved ('slightly improved', 'not changed', 'slightly worsened', 'much worsened' and 'worse than ever').

\section{Secondary outcome measures}

Pain severity of the leg and the back was scored separately on an 11-point numerical rating scale (NRS) ranging from $0=$ no pain to $10=$ unbearable pain [24]. The functional 
status was measured with the Roland Morris Disability Questionnaire (RDQ) for sciatica [20].

Health status was measured by the 36-item short form (SF-36) [27] and the Euroqol (EQ-5D) instrument [4, 5]. Fear of movement was measured by the Tampa scale for kinesiophobia (TSK) [10, 23]. LRS-related absence from work (in days) and medical consumption were measured by means of a questionnaire.

\section{Statistical analysis}

The statistical analysis was performed according to the intention-to-treat principle, analysing all patients in the treatment group to which they were randomly allocated. Baseline comparability was investigated by descriptive statistics to examine whether randomisation was successful. Missing (item) values were assigned the last available score. Group differences and 95\% confidence intervals (CI) were calculated for all outcome measures with a baseline value. Between group differences were calculated using the Student's $t$-test for continuous variables and the Chi-square test for dichotomous variables. Results are presented as relative risks (RR) or effect sizes (ES) with corresponding 95\% CI.

Also presented is the number needed to treat, i.e. the number of patients that needs to be treated, resulting in one more 'improved' patient in the 'GP + PT care' group compared to the 'GP care only' group. There was a statistically significant difference if the $P$-value was smaller than 0.05 , and a clinically relevant difference when a $20 \%$ difference appeared in one of the outcome measurements between both groups. In addition a per-protocol analysis was performed, analysing only those patients with no serious treatment protocol deviations, e.g. received the allocated treatment. Pre-determined subgroup analyses were performed for patients with severe disability (RDQ $\geq 17$ ). Supplementary analysis were performed with multivariate regression to examine the possibility of confounding. The baseline values of variables were used as covariates in the main analyses, whenever appropriate, to adjust for possible differences between the randomised groups.

\section{Results}

\section{Study population}

In total 112 GPs participated in the trial and referred 170 patients for eligibility check. Excluded from the trial were 35 patients for one or more of the following reasons; 16 patients did not want to participate, 1 patient was older than
65 years, 7 patients had (pain) complaints for more than 6 weeks, 3 patients had no radiating (pain) complaints in the leg below the knee, 5 patients were not available for follow-up measurements, 3 patients had back surgery in the past 3 years, 1 patient had received an epidural injection, 7 patients were already treated by a PT and 2 patients were pregnant.

Included and randomised were 135 patients, 67 patients received GP plus PT care (the intervention group) and 68 patients received GP care only (the control group). Four patients dropped-out immediately after randomisation because they no longer wished to participate, one in the intervention group and three in the control group. Figure 2 shows the flow chart of the trial.

\section{Characteristics of the study population}

Table 2 gives the demographic and clinical characteristics of the randomised patients. The two groups were considered comparable for all measured baseline characteristics.

\section{Interventions}

At 6 weeks follow-up, the 67 patients of the intervention group and 68 patients of the control group reported a mean GP consult of, respectively, 1.1 (SD 1.5) and 1.7 (SD 1.8), since baseline.

The mean GP consult at 12 and 52 weeks follow-up was, respectively, 1.6 (SD 1.4) and 1.8 (SD 1.9) in the intervention group and, respectively, 1.9 (SD 2.1) and 2.2 (SD 2.7) in the control group. These differences were not significant. At baseline, the GPs had prescribed NSAIDs, opioids and muscle relaxants for, respectively, 47, 8 and 23 patients in the intervention group, and for, respectively, 40, 10 and 15 patients in the control group.

Patients in the intervention group were treated by 33 different physical therapists. The number of treated patients per physical therapist ranged from 1 to 9 . Patients in the intervention group reported a mean of 6.7 (SD: 2.9) PT treatments of at 6 weeks follow-up, and 9.7 (SD 4.7) at 12 weeks follow-up. The mean time between randomisation and the first PT treatment was 4.6 days (SD 3.1). The physical therapists reported that during the first treatment $60 \%$ (range $10-100 \%$ ) of the time was spent on history taking and physical examination, 30\% (range 0-60\%) on giving information and advice about LRS, and 10\% (range $0-40 \%$ ) on active exercise therapy (duration of one treatment session was $30 \mathrm{~min}$ ). During the second through ninth treatment, $33 \%$ (range $0-100 \%$ ) of the time was spent on giving information and advice, and 67\% (range 0-100\%) on active exercise therapy. 
Fig. 2 Flow chart of the trial

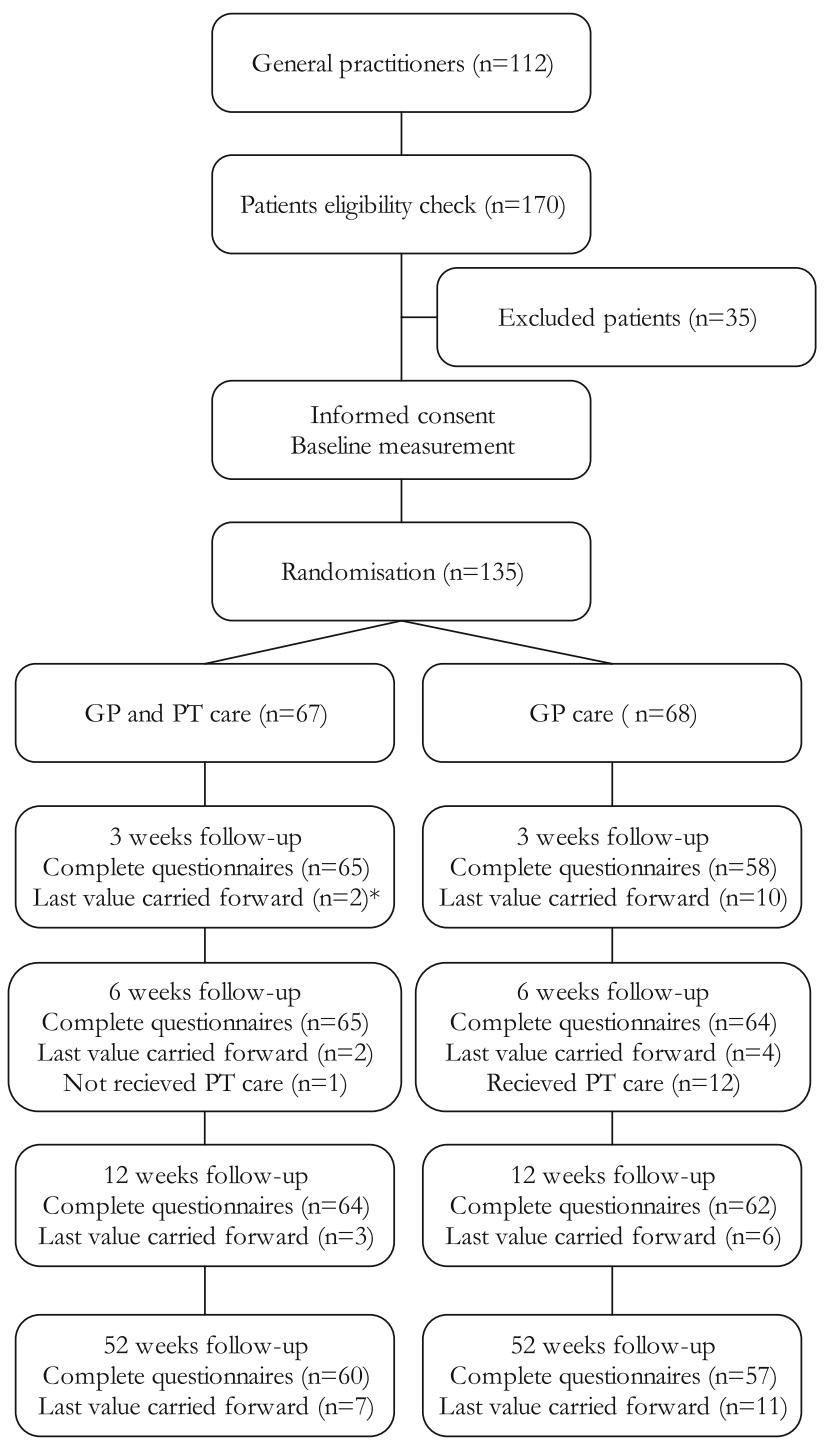

\footnotetext{
*: Last value carried forward is performed for missing values at each follow-up measurement; these include the dropouts ( 1 in the intervention group and 3 in the control group) direct after randomisation plus the number of patients in which some or more data was lacking at each follow-up moment.
}

\section{Outcomes}

In both groups patients improved over time. At 3, 6 and 12 weeks after baseline there was no significant difference between the two groups on the primary outcome: GPE (Table 3). However, at these follow-up moments the intervention group showed a higher proportion of 'improved' patients.

At 52 weeks after baseline there was a significant and a clinical difference between the groups on the primary outcome measure GPE, in favour of the intervention group (Table 3). About 53 patients $(79 \%)$ in the intervention group versus 38 patients $(56 \%)$ in the control group reported to be 'improved' (RR, 1.4; 95\% CI: 1.1; 1.8 and NNT: 4).
There were no significant differences between the groups in most of the secondary outcomes at 3, 6, 12 and 52 weeks after baseline (Table 4).

At 12 and 52 weeks follow-up the mean improvement on leg pain was clinically relevant in both groups; respectively, 3.9 and 4.4 points for the intervention group and, respectively, 3.7 and 3.7 points for the control group. The mean improvement on disability (RDQ) was also clinically relevant at 12 and 52 weeks follow-up in both groups; respectively, 7.7 and 10.0 points for the intervention group and, respectively, 8.5 and 9.1 points for the control group.

The supplementary analyses in which the baseline values were used as covariates, to adjust for possible 
Table 2 Baseline characteristics of the 135 patients randomised in two treatment groups

a NRS Numerical Rating Scale. Score range from 0 (no pain) to 10 (unbearable pain)

${ }^{\mathrm{b}} R D Q$ Roland disability questionnaire. Score range from 0 (no disability) to 24 (severe disability)

c TSK Tampa scale for kinesiophobia. Scores ranges from 17 to 68 points; higher score indicates more kinesiophobia

\begin{tabular}{lll}
\hline Characteristics & $\begin{array}{l}\text { GP }+ \text { PT } \\
\text { care }(n=67)\end{array}$ & $\begin{array}{l}\text { GP care } \\
\text { only }(n=68)\end{array}$ \\
\hline Female gender, $n(\%)$ & $38(57)$ & $27(40)$ \\
Age in years, mean (SD) & $42(10)$ & $43(12)$ \\
Paid job, $n(\%)$ & $48(72)$ & $50(74)$ \\
Reporting sickness absence, $n(\%)$ & $34(51)$ & $32(47)$ \\
Sickness absence from onset in days, mean (SD) & $3.1(4.9)$ & $4.2(6.8)$ \\
Time between onset LRS and baseline in days, mean (SD) & $12.1(10.1)$ & $14.2(10.2)$ \\
Never LRS in past, $n(\%)$ & $49(73)$ & $54(79)$ \\
More pain in leg on coughing, sneezing or straining, $n(\%)$ & $40(60)$ & $37(54)$ \\
Decreased muscle strength in the leg, $n(\%)$ & $48(72)$ & $44(65)$ \\
Sensory deficits in the leg, $n(\%)$ & $54(81)$ & $53(78)$ \\
Positive straight leg raising test, $n(\%)$ & $37(55)$ & $35(52)$ \\
Positive test of Bragard, $n(\%)$ & $25(37)$ & $23(34)$ \\
Taking medication, $n(\%)$ & $58(87)$ & $48(71)$ \\
Leg pain on NRS ${ }^{a}$, mean (SD) & $6.3(2.2)$ & $6.3(2.2)$ \\
Back pain on NRS, mean (SD) & $5.8(2.8)$ & $5.7(2.5)$ \\
RDQb score, mean (SD) & $15.9(4.1)$ & $15.4(5.0)$ \\
TSK $^{\mathrm{c}}$ score, mean (SD) & $39.0(5.8)$ & $41.0(7.1)$ \\
\hline
\end{tabular}

Table 3 Data on treatment results at 3, 6, 12 and 52 weeks after baseline: primary outcome

\begin{tabular}{|c|c|c|c|c|}
\hline Global perceived effect ${ }^{\mathrm{a}}$ & $\begin{array}{l}\mathrm{GP}+\mathrm{PT} \text { care } \\
\text { Improved } n=67(\%)\end{array}$ & $\begin{array}{l}\text { GP care only } \\
\text { Improved } n=68(\%)\end{array}$ & RR $(95 \% \mathrm{CI})$ & NNT \\
\hline Three weeks after baseline & $30(45)$ & $22(32)$ & $1.4(0.9 ; 2.1)$ & 8 \\
\hline Six weeks after baseline & $38(60)$ & $30(44)$ & $1.3(0.9 ; 1.8)$ & 8 \\
\hline Twelve weeks after baseline & $47(70)$ & $42(62)$ & $1.1(0.9 ; 1.5)$ & 12 \\
\hline Fifty-two weeks after baseline & $53(79)$ & $38(56)$ & $1.4(1.1 ; 1.8)$ & 4 \\
\hline
\end{tabular}

${ }^{a}$ Ratings on patient's globally perceived effect on a seven-point scale were dichotomised (see Methods section)

$R R$ Relative risk, $C I$ Confidence interval, $N N T$ Number needed to treat, i.e. the number of patients that needs to betreated, resulting in one more 'improved' patient in the 'GP + PT care' group compared to the 'GP care only' group

differences between the randomised groups showed no changes in the reported results.

There were no significant differences between both groups in the number of patients reporting absence from work or reported days absence from work. At 12 weeks follow-up, 36 patients (54\%) in the intervention group and 25 patients $(37 \%)$ in the control group reported a mean of, respectively, 16.2 days (SD 21.3) and 13.1 days (SD 24.1) absence from work. At 52 weeks follow-up, 30 patients $(45 \%)$ in the intervention group and 25 patients $(37 \%)$ in the control group reported, over the whole year, a mean of, respectively, 29.2 days (SD 48.4) and 28.9 days (SD 72.3) absence from work.

\section{Co-interventions}

In the 52 weeks after baseline 11 patients (16\%) in the intervention group and 6 patients $(9 \%)$ in the control group visited a neurologist. In the intervention group four patients visited a neurosurgeon, one patient a orthopaedist and four patients $(6 \%)$ received surgery due to LRS.

In the control group six patients visited a neurosurgeon, two patients a orthopaedist and three patients (4\%) received surgery. There were a few co-interventions (i.e. occupational physician) in both groups, but there were no significant differences between the two groups.

\section{Per-protocol analysis}

The per-protocol analysis (the patients that received the allocated treatment according randomisation) was restricted to 66 patients in the intervention group and 55 patients in the control group. One patient in the intervention group had not received PT care and 12 patients in the control group were treated by PT. Restricting the analysis to the 
Table 4 Data on treatment results at 3, 6, 12 and 52 weeks after baseline: secondary outcomes

\begin{tabular}{|c|c|c|c|c|}
\hline & $\begin{array}{l}\mathrm{GP}+\mathrm{PT} \text { care } \\
\text { Improvement }(n=67)\end{array}$ & $\begin{array}{l}\text { GP care only } \\
\text { Improvement }(n=68)\end{array}$ & $\begin{array}{l}\text { Mean difference } \\
(\mathrm{GP}+\mathrm{PT})-(\mathrm{GP})(95 \% \mathrm{CI})\end{array}$ & $\begin{array}{l}\text { Effect size } \\
(95 \% \mathrm{CI})\end{array}$ \\
\hline \multicolumn{5}{|c|}{ Three weeks after baseline } \\
\hline Leg pain on $\mathrm{NRS}^{\mathrm{a}, \mathrm{b}}$ & $-2.3(2.4)$ & $-1.9(2.4)$ & $-0.4(-1.2 ; 0.4)$ & $0.17(-0.2 ; 0.5)$ \\
\hline Back pain on $\mathrm{NRS}^{\mathrm{a}, \mathrm{b}}$ & $-2.0(2.8)$ & $-1.7(2.4)$ & $-0.3(-1.2 ; 0.6)$ & $0.12(-0.2 ; 0.5)$ \\
\hline \multicolumn{5}{|l|}{ Six weeks after baseline } \\
\hline Leg pain on $\mathrm{NRS}^{\mathrm{a}, \mathrm{b}}$ & $-3.0(2.7)$ & $-3.3(2.8)$ & $0.3(-0.6 ; 1.2)$ & $0.11(-0.2 ; 0.5)$ \\
\hline Back pain on $\mathrm{NRS}^{\mathrm{a}, \mathrm{b}}$ & $-2.3(3.1)$ & $-2.6(2.7)$ & $0.3(-0.7 ; 1.3)$ & $0.19(-0.2 ; 0.5)$ \\
\hline RDQ score ${ }^{\mathrm{c}, \mathrm{b}}$ & $-5.3(7.0)$ & $-6.6(6.1)$ & $1.3(-0.9 ; 3.6)$ & $0.22(-0.1 ; 0.5)$ \\
\hline General health $^{\mathrm{d}, \mathrm{b}}$ & $2.2(16.4)$ & $-2.8(13.9)$ & $5.0(0.2 ; 10.1)$ & $0.36(-0.0 ; 0.7)$ \\
\hline \multicolumn{5}{|c|}{ Twelve weeks after baseline } \\
\hline Leg pain on NRS ${ }^{\mathrm{a}, \mathrm{b}}$ & $-3.9(2.8)$ & $-3.7(3.1)$ & $-0.2(-1.2 ; 0.8)$ & $0.05(-0.3 ; 0.4)$ \\
\hline Back pain on $\mathrm{NRS}^{\mathrm{a}, \mathrm{b}}$ & $-2.7(3.2)$ & $-2.6(2.9)$ & $-0.1(-1.2 ; 0.9)$ & $0.04(-0.3 ; 0.4)$ \\
\hline RDQ score ${ }^{c, b}$ & $-7.7(7.3)$ & $-8.5(6.7)$ & $0.8(-1.6 ; 3.2)$ & $0.12(-0.2 ; 0.5)$ \\
\hline General health $^{\mathrm{d}, \mathrm{b}}$ & $-1.2(18.4)$ & $-4.7(16.4)$ & $3.5(-2.4 ; 9.5)$ & $0.22(-0.1 ; 0.5)$ \\
\hline \multicolumn{5}{|c|}{ Fifty-two weeks after baseline } \\
\hline Leg pain on NRS ${ }^{a} b$ & $-4.4(2.7)$ & $-3.7(2.7)$ & $-0.7(-1.7 ; 0.2)$ & $0.26(-0.1 ; 0.6)$ \\
\hline Back pain on $\mathrm{NRS}^{\mathrm{a}, \mathrm{b}}$ & $-3.0(3.1)$ & $-2.3(2.9)$ & $-0.7(-1.7 ; 0.4)$ & $0.23(-0.1 ; 0.6)$ \\
\hline RDQ score ${ }^{\mathrm{c}, \mathrm{b}}$ & $-10.0(6.5)$ & $-9.1(6.1)$ & $-0.9(-3.0 ; 1.3)$ & $0.14(-0.2 ; 0.5)$ \\
\hline TSK score ${ }^{\mathrm{b}, \mathrm{e}}$ & $-3.3(7.3)$ & $-4.5(6.6)$ & $1.2(-1.2 ; 3.6)$ & $0.17(-0.2 ; 0.5)$ \\
\hline General health $^{\mathrm{d}, \mathrm{b}}$ & $-3.1(15.7)$ & $-4.1(16.7)$ & $1.0(-4.5 ; 6.5)$ & $0.06(-0.3 ; 0.4)$ \\
\hline
\end{tabular}

All outcome measures are presented in means and standard deviation, unless otherwise stated

${ }^{a}$ NRS Numerical rating scale. Score range from 0 (no pain) to 10 (unbearable pain)

b Negative results denote positive results for patients

c $R D Q$ Roland disability questionnaire. Score range from 0 (no disability) to 24 (severe disability)

${ }^{\mathrm{d}}$ Dimension of the SF-36 = Short form 36 questionnaire. Score range each dimension 0-100; higher score indicates a better health state

e TSK Tampa scale for kinesiophobia. Scores ranges from 17 to 68 points; higher score indicates more kinesiophobia

'per-protocol' patients did not change the within group and between group differences in any substantial way.

\section{Subgroup analysis}

The subgroup with severe disability (RDQ $\geq 17)$ consisted of 67 patients. The intervention group $(n=37)$ and the control group ( $n=30$ ) were considered comparable for all measured baseline characteristics. At 12 and 52 weeks follow-up, respectively, $29(78 \%)$ and $31(84 \%)$ patients with severe disability in the intervention group and $15(50 \%)$ and $16(53 \%)$ patients in the control group reported to be 'improved' (12 and 52 weeks: RR, 1.6; 95\% CI, 1.1; 2.3); indicating a significant and a clinically relevant difference.

\section{Discussion}

At 12 weeks after baseline there was no significant difference between both groups on the primary outcome, but most of the patients $(70 \%$ in the intervention group and
$62 \%$ in the control group) reported to be 'improved'. At 52 weeks follow-up there was a significant (RR, 1.4; 95\% CI, $1.1 ; 1.8)$ and clinically relevant difference $(23 \%)$ between both groups, in favour of the intervention group. However, no significant relevant differences were found in the secondary outcomes: leg pain, functional status, fear of movement and health status. Therefore, adding PT care to GP care is only more effective regarding the primary outcome (GPE) than GP care alone in the long-term, for the average patient with (sub) acute sciatica. Moreover, PT care added to GP care seemed to be especially effective regarding GPE in the subgroup with patients reporting more severe disability at presentation.

Strengths and limitations

Although the control group had three dropouts compared with only one in the intervention group, this did not appear to bias the results because all dropped-out immediately after randomisation, and results of both the per-protocol analysis and the intention-to-treat analysis were similar. 
The loss to follow-up in the study was not very high $(<20 \%)$; at 12 -months follow-up $10 \%(n=7)$ in the intervention group and $16 \%(n=11)$ in the control group. In statistics the missing values were assigned the last available score which could lead to bias towards no differences between the treatment groups, if the loss to follow up is (very) high. However, the per-protocol analysis and the intention-to-treat analysis turned out to be similar.

There are no indications that not blinding GPs, physical therapists and patients biased the results; at 12 weeks follow-up the within-group improvement of both groups was considerable for GPE, the leg pain on NRS and the RDQ.

The trial intended to enrol 182 eligible patients. Finally, 135 patients were randomised in the two treatment groups. For practical reasons (time and money) the recruiting period for patients could not be extended. This may have biased the results, i.e. that the study could have underestimated the effect of the intervention because less patients were randomised according to the sample size calculation. Nevertheless, at 12-months follow-up a significant and clinically relevant difference between both groups, in favour of the intervention group, was found on the primary outcome 'GPE'.

At long-term follow-up a clinically relevant change was found on the primary outcome measure (GPE) between the two groups, in favour of the intervention group; the difference was $23 \%$. A priori was stated that a clinically relevant difference had to be at least 20\% [14]. However, recent work of Ostelo and De Vet shows the need for more research on the exact value for the minimal clinically important difference between two groups in this type of study [17].

\section{Literature}

A few randomised clinical trials have evaluated PT as a treatment for the LRS. Hofstee et al. focused on bed rest, PT and continuation of activities of daily living [8]; their trial included 250 patients with acute sciatica, and the authors concluded after a 6-month follow-up period that bed rest and PT are no more effective than continuation of the activities of daily living [8]. Coxhead et al. compared four methods of PT (traction, exercises, manipulation and corset therapy) in 322 participating patients with sciatica [3]; the authors concluded that although active PT appeared to be of short-term value, it did not seem to confer any long-term benefit [3]. Lidström and Zachrisson compared three methods of PT (massage/exercises, traction and hot packs) in 62 patients with sciatica [11]; after treatment (1 month after randomisation) they concluded that the traction group showed better results than the other two groups [11]. The results of our study are not in concordance with the earlier studies, because we found that in the longterm PT care added to GP care is effective.

There are substantial differences between these clinical trials regarding the study population (e.g. underlying cause of LRS and mix of acute, subacute and chronic patients), control treatments, duration of follow-up, and primary outcome measures. Because of this heterogeneity it is very difficult to compare these studies. Furthermore, the previous studies did not measure the absence from work (or did not report on this outcome). In the present study, there were no significant differences in absence from work between groups at short- or long-term follow-up. The economic evaluation alongside this randomised clinical trial is presented elsewhere [12]. Concluded was that the treatment of patients with LRS with PT and GP care is not more costeffective than GP 'care alone [12].

The results of our study indicate that PT added to GP care was better regarding GPE but not more cost-effective for the average patient with a LRS than GP care alone in the long-term. Moreover, for patients with severe disability at presentation, PT care added to GP care seemed to be especially effective regarding GPE. Future trials are necessary to evaluate the (cost-)effectiveness of PT in patients with severe disability.

Acknowledgements The authors appreciate the assistance of Marie-Louise Lenssinck on patients' management. This trial was funded by the Dutch Health Care Insurance Board (CvZ). An International Standard Randomised Controlled Trial Number was assigned to this trial (ISRCTN68857256) at www.controlled-trials.com.

Open Access This article is distributed under the terms of the Creative Commons Attribution Noncommercial License which permits any noncommercial use, distribution, and reproduction in any medium, provided the original author(s) and source are credited.

\section{References}

1. Bombardier C (2000) Outcome assessments in the evaluation of treatment of spinal disorders: summary and general recommendations. Spine 25:3100-3103

2. Bombardier C, Tugwell P, Sinclair A, Dok C, Anderson G, Buchanan WW (1982) Preference for endpoint measures in clinical trials: results of structured workshops. J Rheumatol 9:798-801

3. Coxhead CE, Inskip H, Meade TW, North WR, Troup JD (1981) Multicentre trial of physiotherapy in the management of sciatic symptoms. Lancet 1:1065-1068

4. Euroqol G (1990) EuroQol-a new facility for the measurement of health-related quality of life. Health Policy 16:199-208

5. Euroqol G (1991) Not a quick fix. Health Serv J 101:29

6. Fries JF (1983) Toward an understanding of patient outcome measurement. Arthritis Rheum 26:697-704

7. Health Council of the Netherlands (1999) Management of the lumbosacral radicular syndrome (sciatica). The Hague:publication no. 1999/1918 
8. Hofstee DJ, Gijtenbeek JM, Hoogland PH, van Houwelingen HC, Kloet A, Lotters F, Tans JT (2002) Westeinde sciatica trial: randomized controlled study of bed rest and physiotherapy for acute sciatica. J Neurosurg 96:45-49

9. Karbowski K (1984) History of the discovery of the Lasegue phenomenon and its variants. Schweiz Med Wochenschr 114:992-995

10. Kori SH, Miller RP, Todd DD (1990) Kinesophobia: a new view of chronic pain behaviour. Pain Manage Jan/Feb:35-43

11. Lidström A, Zachrisson M (1970) Physical therapy on low back pain and sciatica. An attempt at evaluation. Scand J Rehabil Med 2:37-42

12. Luijsterburg PAJ, Lamers LM, Verhagen AP, Ostelo RWJG, van den Hoogen HJMM, Peul WC, Avezaat CJJ, Koes BW (2007) Cost-effectiveness of physical therapy and general practitioner care for sciatica. Spine 32:1942-1948

13. Luijsterburg PAJ, Verhagen AP, Braak S, Oemraw A, Avezaat CJJ, Koes BW (2005) General practitioners' management of LRS compared with a clinical guideline. Eur J Gen Pract 11:113-121

14. Luijsterburg PAJ, Verhagen AP, Ostelo RWJG, van den Hoogen JMM, Peul WC, Avezaat CJJ, Koes BW (2004) Conservative treatment in patients with an acute lumbosacral radicular syndrome: design of a randomised clinical trial. BMC Musculoskelet Disord 5:39

15. Luijsterburg PAJ, Verhagen AP, Ostelo RWJG, van Os TA, Peul WC, Koes BW (2007) Effectiveness of conservative treatments for the lumbosacral radicular syndrome: a systematic review. Eur Spine J 16:881-899

16. Mens JMA, Chavannes AW, Koes BW, Lubbers WJ, Ostelo RWJG, Spinnewijn WEM, Kolnaar BGM (2005) [NHG-guideline Lumbosacral Radicular Syndrome] NHG-Standaard Lumbosacraal Radiculair Syndroom. Huisarts en Wetenschap 48:171-178

17. Ostelo RWJG, de Vet HC (2005) Clinically important outcomes in low back pain. Best Pract Res Clin Rheumatol 19:593-607

18. Ostelo RWJG, de Vet HC, Waddell G, Kerckhoffs MR, Leffers P, van Tulder MW (2003) Rehabilitation following first-time lumbar disc surgery: a systematic review within the framework of the cochrane collaboration. Spine 28:209-218

19. Roberts C, Torgerson D (1998) Randomisation methods in controlled trials. BMJ 317:1301

20. Roland M, Morris R (1983) A study of the natural history of back pain. Part I: development of a reliable and sensitive measure of disability in low-back pain. Spine 8:141-144

21. Smeele IJM, Van den Hoogen JMM, Mens JMA, Chavannes AW, Faas A, Koes BW, Romeijnders ACM, Van der Laan JR (1996) [NHG-guideline Lumbosacral Radicular Syndrome] NHGStandaard Lumbosacraal Radiculair Syndroom. Huisarts en Wetenschap 39:78-89

22. Stam J (1996) Consensus in diagnosing and treatment of the lumbosacral radicular syndrome. Consensus over diagnostiek en behandeling van het lumbosacrale radiculaire syndroom. Ned Tijdschr Geneeskd 140:2621-2627

23. Vlaeyen JW, Kole-Snijders AM, Boeren RG, van Eek H (1995) Fear of movement/(re)injury in chronic low back pain and its relation to behavioral performance. Pain 62:363-372

24. Von Korff M, Jensen MP, Karoly P (2000) Assessing global pain severity by self-report in clinical and health services research. Spine 25:3140-3151

25. Vroomen PC, de Krom MC, Slofstra PD, Knottnerus JA (2000) Conservative treatment of sciatica: a systematic review. J Spinal Disord 13:463-469

26. Vroomen PC, de Krom MC, Wilmink JT, Kester AD, Knottnerus JA (1999) Lack of effectiveness of bed rest for sciatica. N Eng J Med 340:418-423

27. Ware JE Jr, Sherbourne CD (1992) The MOS 36-item short-form health survey (SF-36). I. Conceptual framework and item selection. Med Care 30:473-483

28. Weber H, Holme I, Amlie E (1993) The natural course of acute sciatica with nerve root symptoms in a double-blind placebocontrolled trial evaluating the effect of piroxicam. Spine 18:1433-1438 